\title{
EchoGéo
}

$50 \mid 2019$

Varia

\section{Questions sociales, questions urbaines : questions de géographie}

Éditorial

Jean-Louis Chaléard

\section{OpenEdition}

Journals

Édition électronique

URL : https://journals.openedition.org/echogeo/18361

DOI : 10.4000/echogeo.18361

ISSN : 1963-1197

Éditeur

Pôle de recherche pour l'organisation et la diffusion de l'information géographique (CNRS UMR 8586)

\section{Référence électronique}

Jean-Louis Chaléard, «Questions sociales, questions urbaines : questions de géographie », EchoGéo [En ligne], 50 | 2019, mis en ligne le 31 décembre 2019, consulté le 01 août 2021. URL : http:// journals.openedition.org/echogeo/18361 ; DOI : https://doi.org/10.4000/echogeo.18361

Ce document a été généré automatiquement le 1 août 2021.

EchoGéo est mis à disposition selon les termes de la licence Creative Commons Attribution - Pas d'Utilisation Commerciale - Pas de Modification 4.0 International (CC BY-NC-ND) 


\title{
Questions sociales, questions urbaines : questions de géographie
} Éditorial

\author{
Jean-Louis Chaléard
}

1 Faut-il revenir encore sur ce qui apparaît comme une tendance lourde: la prépondérance de l'urbain dans les sujets abordés? En effet, la présente livraison d'EchoGéo, comme la plupart des précédents Varia, propose des contributions qui touchent à la ville. L'environnement constitue le deuxième champ d'études majeur du numéro, en retrait par rapport au précédent. Sans doute faut-il voir dans la prépondérance de ces thèmes, le reflet de notre monde caractérisé par son urbanisation accélérée, le fait que la majorité des habitants de la terre vit aujourd'hui dans les villes, et la montée des inquiétudes environnementales face aux risques naturels variés qui menacent notre quotidien. Ces thèmes illustrent la capacité de la géographie à se situer au cœur des questions contemporaines. Ils inscrivent également la discipline dans des débats scientifiques où celle-ci est confrontée à d'autres sciences de la société, pouvant faire valoir son approche, ses interrogations, ses résultats.

2 La rubrique Sur le champ propose quatre articles qui posent plus ou moins selon les textes des questions sociales et/ou d'aménagement urbain, tout en s'inscrivant dans des branches différentes de la géographie et se confrontant à d'autres disciplines. Le premier article aborde "les mères de jumeaux en situation de mendicité à Ouagadougou ". Le sujet semble relever d'abord de la sociologie et renvoie à des interrogations sur la précarité et la vulnérabilité. Au-delà, les autrices, A. Nikiema et H.-P. Sawadogo, mettent en évidence une géographie de la mendicité en relation avec des pratiques culturelles et la situation économique des mères. En effet, si la gémellité a une signification particulière dans les systèmes de représentation des groupes socioculturels au Burkina Faso, et plus largement en Afrique subsaharienne, elle est aujourd'hui détournée dans une mendicité aux motifs économiques. A. Nikiema et H.P. Sawadogo décrivent les sites de mendicité préférentiels occupés par les femmes et cherchent à comprendre la logique d'occupation de ces lieux. Au-delà, elles relèvent l'évolution de l'espace urbain. Les sites les plus fréquentés se situent dans la première 
couronne périphérique lotie entre les années 1980 et 1990 et dans une moindre mesure dans le centre-ville, marqués par la forte présence de services et le dynamisme des échanges économiques.

3 Le deuxième texte porte sur les « jardins familiaux », autrefois « jardins ouvriers ", en Île-de-France, objet de nombreuses études depuis leur apparition à la fin du XIXe siècle, dans des disciplines variées, et qui soulèvent des enjeux à la fois urbanistiques, alimentaires et sociaux. L'article de C. Robert-Bœuf s'interroge sur leur participation à la fabrique de la ville et leur relatif maintien dans un milieu urbain où les espaces ouverts s'amenuisent. Ces jardins s'inscrivent dans l'espace urbain en articulant plusieurs échelles et différents types d'acteurs. Participant originellement à l'urbanisation des banlieues, ils en sont aujourd'hui les victimes. Cependant, plus qu'une végétalisation de la ville et une amélioration du cadre de vie des urbains, ils favorisent, selon l'autrice, une « agrarisation » de la métropole de plus en plus discutée.

4 L'article de A. R. Mejía Uquiche et J. Ronchail aborde la question des risques urbains, à travers l'étude des écoulements torrentiels à Chosica, en périphérie de Lima, sur les contreforts de Andes, fréquemment frappée par des écoulements torrentiels. S'appuyant sur les cas des écoulements de 2012, 2015 et 2017, les auteurs analysent successivement l'aléa torrentiel, la vulnérabilité de la population, comprise comme la susceptibilité à subir des dommages, et la politique de gestion du risque, absente des politiques publiques pendant longtemps à Lima. Ces événements ne sont pas seulement liés à l'occurrence de pluies fortes, mais aussi aux conditions d'instabilité géomorphologique dans des ravins, dont les parties inférieure et centrale sont peuplées. Ils entraînent de fortes pertes en vies humaines et des dommages économiques importants. Les auteurs soulignent, au-delà des retards et des difficultés à mener une politique préventive, que l'installation de barrières flexibles contribue à la réduction des dégâts, signe que des solutions existent, qui permettent d'amoindrir les effets des écoulements.

Le dernier article du Varia relève de la géographie de la santé. M. Jarraya étudie la météopathologie des conjonctivites à Sfax, en Tunisie. Il met en évidence que la morbidité liée aux conjonctivites est fortement influencée par le contexte climatique. S'appuyant sur des données chiffrées et des enquêtes auprès des médecins et des malades, il montre que l'accroissement de la morbidité en hiver et au printemps est lié à des facteurs différents : conjonctivite d'origine infectieuse en hiver, allergiques au printemps, mais toutes en relation directe ou indirecte avec les conditions climatiques. Comme pour l'article précédent, les analyses débouchent sur des conclusions opérationnelles visant à améliorer la situation des populations touchées ou à risque, offrant ainsi des éléments pour une véritable géographie appliquée.

6 Des articles de géographie électorale alimentent régulièrement la rubrique Sur le vif, soulignant le dynamisme d'un champ à la rencontre de la géographie et des sciences politiques. M. B. Timera, M. Diongue et O. Thiam (parution fin 2019) abordent les résultats des élections présidentielles qui ont eu lieu le 24 février 2019 au Sénégal et qui ont vu la victoire du président sortant, Macky Sall. Le cas sénégalais est particulièrement intéressant parce que le pays est souvent présenté comme un modèle de démocratie en Afrique ou, à tout le moins, un pays pleinement entré dans le jeu de la démocratie représentative. Dès lors, les débats et les contestations qui ont eu lieu avant et après le scrutin prennent une résonance particulière. Les auteurs analysent le contexte de l'élection marqué, entre autres, par l'éviction, au nom de la lutte contre la 
corruption, de candidats potentiels, la réduction du nombre de prétendants en raison de nouvelles exigences pour se présenter, la défection des deux grands partis historiques (Parti socialiste et Parti démocratique sénégalais)... Ils soulignent les tendances nouvelles de la géographie électorale avec un poids plus sensible du vote régional ou régionaliste. Pour finir, ils s'interrogent sur l'avenir dans un jeu politique caractérisé par l'imprévisibilité et l'instabilité.

$7 \quad$ Le second article de la rubrique Sur le vif est aussi à forte connotation politique et fait écho aux mobilisations sociales qui agitent la planète actuellement, du Chili à la France, en passant par le Soudan, l'Ukraine et bien d'autres pays (parution début 2020). J.M. Théodat analyse les mouvements qui ont affecté Haïti de juillet 2018 à novembre 2019 et qui se poursuivent encore aujourd'hui. S'ils peuvent être inscrits dans une vague plus générale d'agitation et de contestation de l'ordre social et des gouvernements en place, l'auteur insiste sur la singularité du cas haïtien dans un pays marqué par une extrême pauvreté, un sous-équipement dramatique et une polarisation du territoire par des gangs qui s'arrogent le droit de lever des taxes et de percevoir des dimes sur les civils dans les quartiers insalubres et d'habitat précaire où la police ne se hasarde plus. Dans une société en miette, et dans un contexte de discrédit des partis politiques traditionnels et d'une opposition réduite à "des groupuscules partisans ", ces mouvements marquent, selon l'auteur, la fin du citoyen au profit de l'individu, branché sur internet et les réseaux sociaux. Dès lors, pour lui, un changement de régime nécessite une révision en bloc du fait même de gouverner.

8 Ainsi, le tropisme urbain de ce numéro n'empêche pas une diversité qui tient aux approches et à la nature des sujets, permettant d'alimenter des débats nombreux, de l'aménagement du territoire à des crises sociales multiformes. 